EUROPEAN ORGANIZATION FOR NUCLEAR RESEARCH

European Laboratory for Particle Physics

Large Hadron Collider Project

LHC Project Report 497

\title{
METHODS AND RESULTS OF MODELING AND TRANSMISSION-LINE CALCULATIONS OF THE SUPERCONDUCTING DIPOLE CHAINS OF CERN'S LHC COLLIDER
}

F. Bourgeois and K. Dahlerup-Petersen

\begin{abstract}
Electrical modeling and simulation of the LHC magnet strings are being used to determine the key parameters that are needed for the design of the powering and energy extraction equipment.

Poles and zeros of the Laplace expression approximating the Bode plot of the measured coil impedance are used to synthesize an R/L/C model of the magnet. Subsequently, this model is used to simulate the behavior of the LHC main dipole magnet string. Lumped transmission line behavior, impedance, resonance's, propagation of the power supply ripple, ramping errors, energy extraction transients and their damping are presented in this paper.
\end{abstract}

LHC Division.

Presented at the 13th International Pulsed Power Conference (PPPS2001)

17-22 June 2001, Las Vegas, Nevada, USA

\footnotetext{
Administrative Secretariat

LHC Division

CERN

CH - 1211 Geneva 23

Switzerland

Geneva, 27 August 2001
} 


\title{
METHODS AND RESULTS OF MODELING AND TRANSMISSION-LINE CALCULATIONS OF THE SUPERCONDUCTING DIPOLE CHAINS OF CERN's LHC COLLIDER
}

\author{
F. Bourgeois and K. Dahlerup-Petersen \\ CERN, Geneva, Switzerland
}

\begin{abstract}
Electrical modeling and simulation of the LHC magnet strings are being used to determine the key parameters that are needed for the design of the powering and energy extraction equipment.

Poles and zeros of the Laplace expression approximating the Bode plot of the measured coil impedance are used to synthesize an R/L/C model of the magnet. Subsequently, this model is used to simulate the behavior of the LHC main dipole magnet string. Lumped transmission line behavior, impedance, resonance's, propagation of the power supply ripple, ramping errors, energy extraction transients and their damping are presented in this paper.
\end{abstract}

\section{Introduction}

Magnet modeling and transmission line studies awake interest among accelerator design specialists from many different disciplines. Resonance frequencies and the impedance of the magnet string are of prime importance for operational purposes. The lowfrequency tune modulation caused by the power converter ripple is responsible for reducing the dynamic aperture in presence of non-linear fields. For the energy extraction the transient voltages, occurring during opening of the dump switches, may result in excessive stressing of the dielectrics. The need for additional, individual damping across each magnet is determined at the same occasion. Excellent tracking of the ideal current throughout the magnet string is of considerable importance for the beam stability. Finally, the results of the study are used for the determination of important features of the power circuit, such as the leakage current through the grounding resistor, the consequences of the choice of grounding point and the usefulness of transmission line terminations. The answers to all these vital questions can be addressed as soon as the first magnet is available, by elaboration of a synthesized electrical model and numerical simulation of series-connected magnet models.

\section{Electrical modeling and lumped transmission line behavior of the LHC dipoles string}

\section{A. Introduction}

This section is a short account of the synthesis methodology used for the electrical modeling of a passive device into an $\mathrm{R} / \mathrm{L} / \mathrm{C}$ circuit. Amplitude and phase measurements of the magnet under test are used to find sets of poles and zeros approximating its electrical behavior through the whole frequency bandwidth. An electrically compatible R/L/C model can then be synthesized by means of the combination of two conventional algorithms. It is then shown that the LHC dipole string behaves as a 3-km long lumped transmission line with $25 \mathrm{~ms}$ propagation delay.

B. Basic expression of a passive impedance

Passive impedance synthesis is an old problem, which has lost relevance since the advent of active digital filters. It is of interest for simulation purposes when the electrical characteristics of a passive device are not known.

The commonly used mathematical expression of passive impedance is a fraction with polynomials of the Laplace variable s at numerator and denominator. Such a passive impedance fraction must satisfy the following requirements:

- Polynomial coefficients are positive $\&$ real ;

- There is no pole or zero at $\operatorname{Re}(s)>0$;

- The number of zeros differs by less than two units from the number of poles ;

- There is at most one pole (or zero) at origin (or infinity);

\section{Electrical modeling of a magnet}

\section{Methodology}

To find an electrically equivalent model of a real magnet it is common practice to measure the voltage appearing across the magnet coil as a function of frequency, when it is driven by a current source of known amplitude. This measurement of the amplitude (in decibels) and the phase (in degrees) of the impedance of the device under test can be easily achieved with the help of a Transfer Function Analyzer TFA [1]. This results in the Bode plot shown in Figure 1 (modulus of the impedance, roof shaped curve peaking at $4 \mathrm{kHz}$ for the LHC superconducting main dipole magnet).

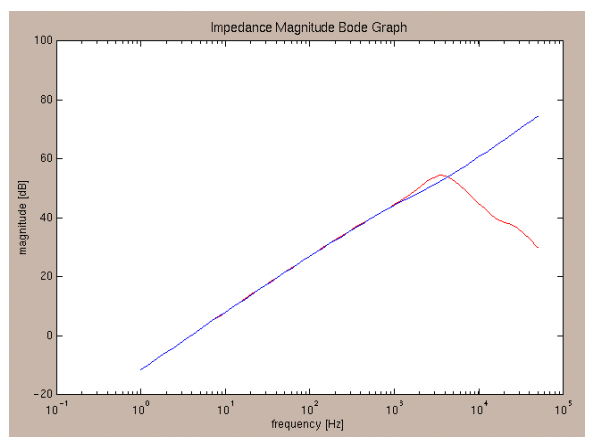

Figure 1: Raw data and first 2-pole, 3-zero fit

So far, the conventional modeling method has been to guess an $\mathrm{R} / \mathrm{L} / \mathrm{C}$ electrical model of the magnet and then to assign component values giving a good 
approximation of the measured Bode plot. The main trouble with this method is that a change in the value of a component can affect the whole plot. Poles and zeros change place and phase can be loosely approximated. It is in some respect a trial and error methodology, which requires expertise.

It was therefore found more appropriate:

1. To model the analytic expression of the impedance function, and then

2. To synthesize it as a passive circuit (if ever necessary since the Laplace expression can be readily used by a large number of simulation tools!).

\section{Fitting the TFA measurements}

The first step consists of a sensible tracking of the Bode plot by means of an iterative process, basically an interactive fit of the amplitude and phase curves. At the $i^{\text {th }}$ iteration a new pole (or a new zero, or a new pair of imaginary conjugates) is added so that the low frequency part of the fit is not affected.

Let $\omega$ denote the pulsation and $\overline{\text { its imaginary }}$ conjugate. Four types of increments can be used at the $i^{\text {th }}$ iteration and their Laplace expressions are:

$\left(\mathrm{s}+\omega_{\mathrm{i}}\right) / \omega_{\mathrm{i}}$ or $\omega_{\mathrm{i}} /\left(\mathrm{s}+\omega_{\mathrm{i}}\right)$ or $\left[\omega_{\mathrm{i}} /\left(\mathrm{s}+\omega_{\mathrm{i}}\right)+\bar{\omega}_{\mathrm{i}} /\right.$ $\left.\left(\mathrm{s}+\bar{\omega}_{\mathrm{i}}\right)\right]^{ \pm 1}$

It is readily seen that the modulus of these fractions is equal to 1 for values of the Laplace variable s smaller than $j \omega_{i}$. Hence, adding such a function will not significantly affect the first part of the achieved fit, say up to $0.5^{*} \omega_{1}$.

Figure 1 shows this process for the LHC main dipole magnet at the fifth iteration when 2 poles and 3 zeros have been assigned. To streamline the tracking, the Bode fit program (written in Matlab ${ }^{\mathrm{TM}}$ ) displays amplitude and phase values in pairs of plots giving the absolute value and the difference between the fit and the TFA measurements (bounded at $3 \mathrm{~dB}$ and $45^{\circ}$ respectively). It is thus easy to find the next $\omega_{1}$ value and pursue the fit to the highest frequency of interest.

The [6-pole, 6-zero] approximation used for the simulations presented in this paper is shown in Figure 2. Errors are well within $\pm 3 \%$ in amplitude $(0.3$

${ }^{\circ}$ in phase. This was compared to a refined [12-pole, 11-zero] approximation, which did not give significantly better results.

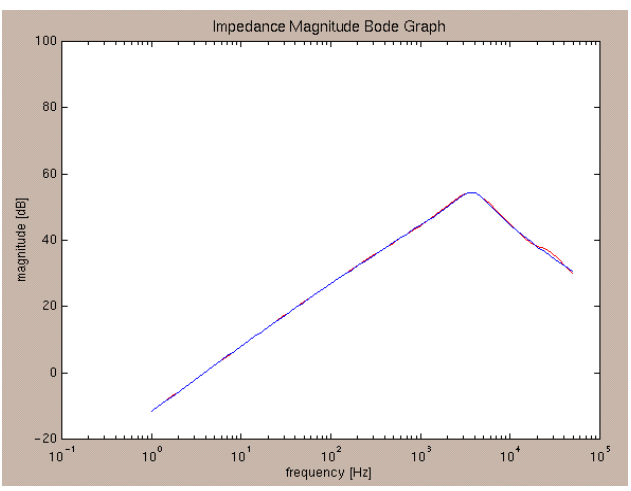

Figure 2: 6-pole, 6-zero fit of the Bode plot

\section{From poles and zeros to the $R / L / C$ model}

Impedance synthesis algorithms can be found in [2],[3]. There exist a (very) large number of equivalent passive circuits. Some are very remote from the physical circuit. Brune cells $(\mathrm{R} / \mathrm{L} / \mathrm{C})$, Foster $(\mathrm{L} / \mathrm{C})$, Cauer $(\mathrm{R} / \mathrm{C})$ and $\mathrm{L} / \mathrm{C}$ four-pole terminated by a resistor synthesis can be used. However, it was found more practical to use a direct decomposition of the impedance fraction in the form of a continued fraction. This decomposition consists of expressing the impedance as $\mathrm{Z}=\mathrm{Z} 0+1 /(\mathrm{Y} 1+1 /(\mathrm{Z} 2+$ etc $)$. Thus, after a series of polynomial divisions, the fraction $Z=\left(8 s^{3}+30 s^{2}+37 s+10\right) /\left(4 s^{2}+9 s+3\right)$ can be written $\mathrm{Z}=2 \mathrm{~s}+3+(1 /(\mathrm{s}+2+1 /(4 \mathrm{~s}+1))$. This decomposition corresponds to: ( (a $4 \mathrm{H}$ inductor in series with a $1 \Omega$ resistor) in parallel with a $1 \mathrm{~F}$ capacitor in parallel with a $1 / 2 \Omega$ resistor) in series with a $2 \mathrm{H}$ inductor in series with a $3 \Omega$ resistor.

The continued fraction decomposition can unfortunately result in the assignment of negative component values. Engineers do not easily admit this idiosyncrasy even though such negative values are not violating the passive impedance criteria and can be properly used by simulators.

In order to minimize the number of negative component values and to build a palette of equivalent passive impedance circuits, the continued fraction algorithm has been combined with decomposition in "residues". To begin with, the impedance fraction is decomposed as $\mathrm{Z}=\mathrm{Z}_{0}+\sum \mathrm{a}_{\mathrm{i}} /\left(\mathrm{s}+\omega_{\mathrm{i}}\right)$. Where the $\omega_{\mathrm{i}}$ are the $\mathrm{n}$ poles of the impedance fraction, and $\mathrm{Z}_{0}$ is the remainder of the decomposition (order $<2$ ). The program then searches for all combinations of subsets of $\mathrm{p}<\mathrm{n}$ residues which can be synthesized by the continued fraction algorithm without giving negative component values. It is then possible to select the solution that best match the physical characteristics of the magnet. This method gives a large number of satisfactory solutions in a few minutes (Matlab ${ }^{\mathrm{TM}}$ on Sun $^{\mathrm{TM}}$ ).

\section{Final modeling steps}

The impedance synthesis program is a necessary tool to establish a satisfactory electrical model of the magnet. The reason stems from the fact that the coil impedance model must be combined with the coil to ground impedance model (in first approximation, the capacitance of the coil with respect to ground) [1]. Thus, to come to the $\mathrm{LHC}$ main dipole $\mathrm{R} / \mathrm{L} / \mathrm{C}$ model of Figure 3, the authors had to process two TFA measurements, to run twice the synthesis program, and last to merge these two models. The latter is straightforward since some components have values that are orders of magnitude bigger/smaller than others. In addition, it was not possible to perform TFA measurements below $1 \mathrm{~Hz}$. As the DC inductance obtained from energy measurements, made by CERN/LHC/MTA group during slow current ramping, was found to be $104 \mathrm{mH}$ up to approximately $0.05 \mathrm{~Hz}$, it was decided to add a pole/zero pair at $0.05 / 0.063 \mathrm{~Hz}$ to complete the model. Figure 3 corresponds to the full model, valid from $0.01 \mathrm{~Hz}$ to $50 \mathrm{kHz}$. 


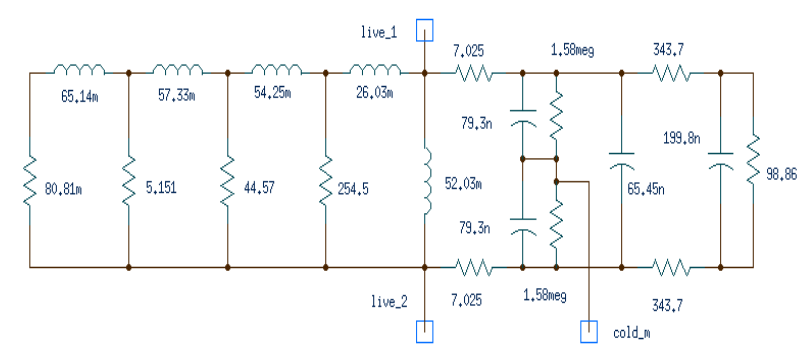

Figure 3: LHC main dipole passive component model

\section{The LHC dipole chain as a lumped transmission line}

For radio frequency (RF) engineers, a transmission line is a pair of conductors with constant cross section. It is well known that such a line is seen as a pure resistor by the generator (infinite line). This equivalent resistor is the characteristic impedance $Z_{c}$ of the line. Signals propagate along the line with a propagation delay / unit length $\mathrm{t}_{\mathrm{pd}}=(\varepsilon \mu)^{1 / 2}$ where $\varepsilon$ and $\mu$ are the permitivity and the permeability of the medium in between the two conductors. In a finite transmission line a fraction $\rho=\left(Z_{t}-Z_{c}\right) /\left(Z_{t}+Z_{c}\right)$ of the incoming signal will be reflected at the end of the line if it is terminated by $\mathrm{Z}_{\mathrm{t}} \neq \mathrm{Z}_{\mathrm{c}}$. Standing waves (RF) and pulse clipping effects are a direct consequence of the reflections.

A lumped transmission line is a series of $\mathrm{L} / \mathrm{C}$ cells and it behaves very much like a pure transmission line. The characteristic impedance of a lumped line is $\mathrm{Z}_{\mathrm{c}}=$ $(\mathrm{L} / \mathrm{C})^{1 / 2}$ and the propagation delay per cell $t_{\mathrm{pd}}=(\mathrm{L} . \mathrm{C})^{1 / 2}$. It can be seen from Figure 3 that above $1 \mathrm{~Hz}$ for one aperture of the LHC main dipole $\mathrm{L}=41.34 \mathrm{mH}, \mathrm{C}=$ $158 \mathrm{nF}$. These figures give a $\mathrm{t}_{\mathrm{pd}}$ of $80.82 \mu$ s per aperture or $24.9 \mathrm{~ms}$ for the 154-dipole string. The characteristic impedance is $Z_{c}=511 \Omega$ and it will be seen in the following that most simulation results can be crosschecked against these basic two parameters.

The simulation of the propagation of a $1 \mathrm{~A}, 10 \mathrm{~ms}$ current pulse in a magnet string terminated by a low impedance to ground ( $1 \Omega$, LHC case) is shown in Figure 4 to illustrate a negative reflection $(\rho=-1)$.

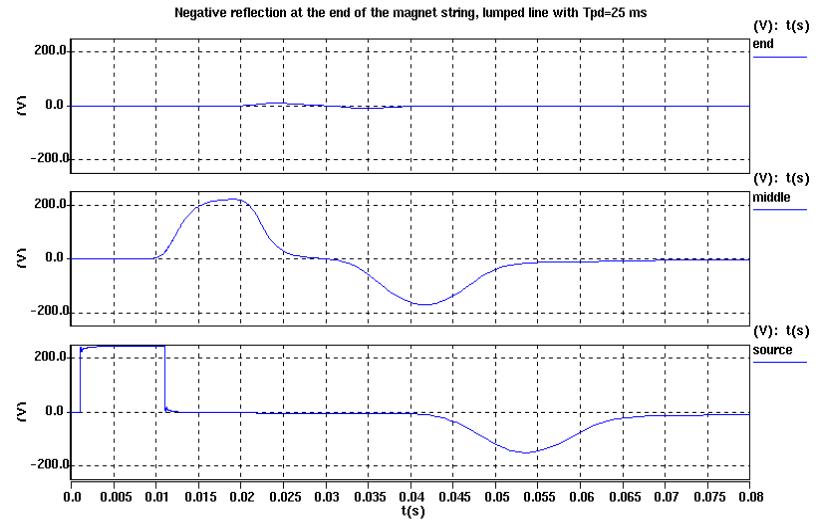

Figure 4: Reflection of a 1A, $10 \mathrm{~ms}$ current pulse

It results from this $25 \mathrm{~ms}$ propagation delay that the fundamental modes of oscillations of this 154 dipole string are $10 \mathrm{~Hz}$ and its multiples. Shorter chains will, therefore, exhibit fundamental modes in inverse proportion to the number of magnets.

\section{Simulation results}

The power circuit used for the simulations is shown in Figure 5.

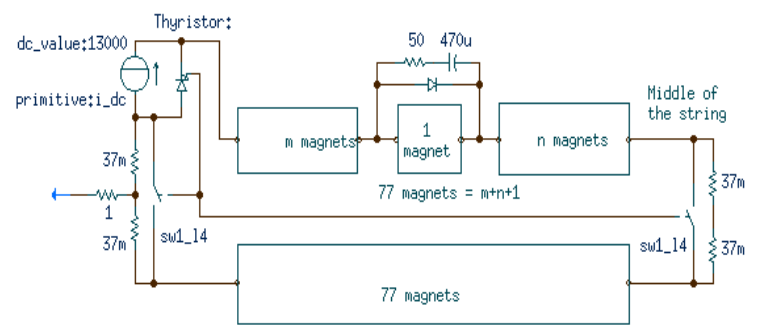

Figure 5. Power circuit, earthing through $1 \Omega$ in either midpoint of the dump resistors.

\section{A. Impedance spectrum}

Amplitude diagrams for the undamped load impedance (a complete chain of 154 dipoles) are shown in Figure 6. Three configurations are shown: grounding next to the power supply, grounding at the end of the string (mid-point of the transmission line), same as the latter but with $511 \Omega$ terminations at each power supply terminal. As can be inferred from the lumped line characteristics, first resonance is at $10 \mathrm{~Hz}$ and $20 \mathrm{~Hz}$ respectively.

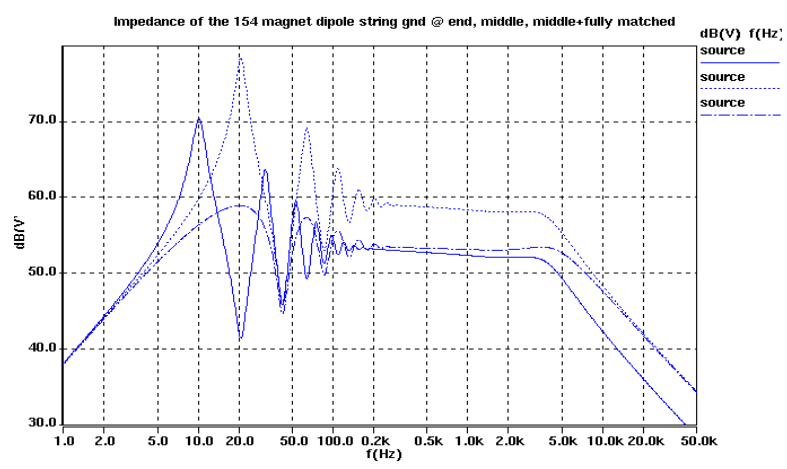

Figure 6: Impedance of the LHC main dipole sting for end-grounding (full line), center-grounding (dotted line) and a fully matched lumped line with 2 × $511 \Omega$ (semi-dotted line). Center-grounding results in higher impedance $(6 \mathrm{~dB})$. Matching stabilizes the impedance throughout the frequency bandwidth.

\section{B. Ripple Analysis}

The magnet current ripple will depend on frequency and the position of the magnet in the string. The location of the grounding point and the individual magnet damping will influence the current ripple value to a smaller extent. As shown in Figure 7 and in first approximation, the current ripple is equal to the power supply voltage ripple divided by the characteristic impedance of the line $(511 \Omega)$. At the two extremities of the magnet chain the ripple is closely related to the impedance plot. Inside the chain the ripple is 
significantly attenuated above $200 \mathrm{~Hz}$. Thus, for $\Delta \mathrm{U}=$ $200 \mathrm{mV}_{\text {peak-peak }}$ and a normalized ripple of $\mathrm{r}=-41.6 \mathrm{~dB}$ :

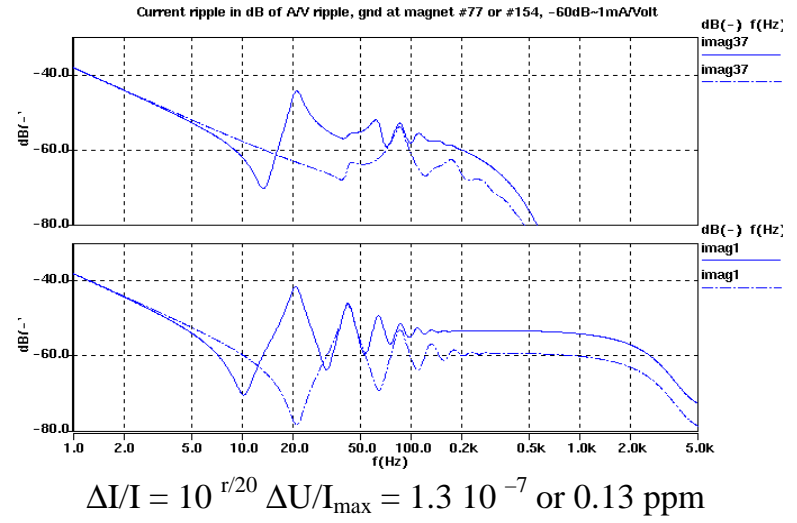

Figure 7: Current ripple at beginning and first quarter of the magnet string. For center (dashed line) and end grounded (plain line) circuit.

The center-grounded circuit features lower ripples, except for the last magnet in each sub-chain near the center extraction system, where there is no difference.

\section{Ramping}

By application of various forms of current ramps, the model has been used to calculate the instantaneous differences in the currents between the individual dipoles during the ramping process

It was found that the center-grounded system results in a maximum deviation $50 \%$ smaller than that of the end grounded arrangement. The largest errors occur at the end of each half-chain, typically $24 \mathrm{~mA} / 13000 \mathrm{~A}$ or $2 \mathrm{ppm}$ in the case of end-earthing.

\section{Transient effects.}

The study of voltage wave propagation in the magnet chains is of particular interest for determination of the dielectric stresses occurring upon opening of the energy extraction switches. It is here that individual, additional damping of the superconducting magnets becomes an important issue. By simultaneous opening of the two switch systems, voltage waves of opposite polarity will travel into each half-chain from each end, the initial amplitudes being equal to half the voltage rise across the dump resistors. The waves will be naturally damped in the magnet chains after a few $100 \mathrm{~ms}$ and their addition will result in local oscillations. In this case additional damping of each magnet is an efficient way to reduce the magnitude of the problem (see Figure 5). However, the unavoidable delay between the opening of the switches of the two systems will worsen the situation. The switches, being fast mechanical breakers, operate by both a pulsed release and an under-voltage release. Normally, the pulsed trigger will open the switches in $5+/-1 \mathrm{~ms}$.. However, if one system fails to open in the pulsed mode, the opening time difference will be $15 \mathrm{~ms}$.. In this case, where the delay is comparable to the wave travelling time through half the dipole chain $(12.5 \mathrm{~ms})$, the oscillations are difficult to damp as shown in Figure 8 .

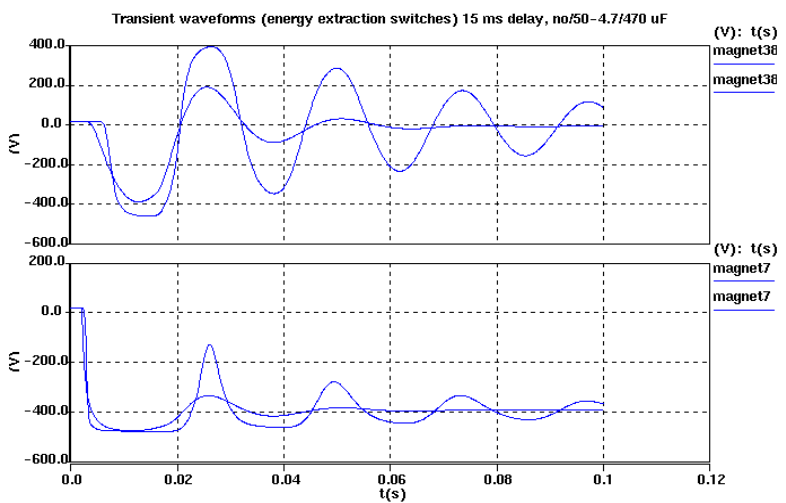

Figure 8: Damped and un-damped oscillations occurring in case of failure of fast (pulsed) opening of one breaker system

There are two reasons to apply additional damping by a $\mathrm{RC}$ circuit:

1. During ramping the current, bypassing the magnet, will only originate from voltage variations (and be zero during a linear part of the current ramp) and

2. The cold protection diode across each magnet needs a snubber circuit for over voltage transient limitation.

However, combined with a typical damping resistance of $70 \Omega$, the capacitance will reduce the damping if the break frequency is too low. This issue requires careful optimisation.

\section{Conclusions}

For the magnet modeling: $\mathrm{R} / \mathrm{L} / \mathrm{C}$ synthesis is needed to combine different TFA measurements. . Simplified $\mathrm{R} / \mathrm{L} / \mathrm{C}$ magnet models can easily be checked against sophisticated ones. With the gained experience, most magnet strings can be synthesized and thoroughly simulated in just a few days

For the LHC application: the transmission-line approach provides answers to numerous questions from many different fields. The above results are a small part of the available data and other LHC magnet strings will be studied. Furthermore, a similar analysis with a superimposed DC current is planned.

\section{ACKNOWLEDGEMENTS}

The authors wish to thank Dr. Oleg Kurnaev, IHEP, Protvino for many stimulating discussions on the issue.

\section{REFERENCES}

[1] K. Dahlerup-Petersen and F. Schmidt, CERN, Geneva, Switzerland: "Evaluation of Impact from Ripple and Transient Phenomena in the LHC Dipole Srings - Methods and Results", EPAC 1996.

[2] Balabanian, Norman ; Bickart, Theodore A \& Seshu, Sundaram: "Electrical Network Theory" p. 467570 ; John Wiley \& Sons Inc, New York (C) 1969

[3] Hasler, Martin ; Neirynck, Jacques: "Electric filters" ; Artech House, Dedham, MA, (C1986 